\title{
HIGHLIGHTS
}

ACUTE CORONARY SYNDROMES

\section{New blood tests improve risk assessment and early diagnosis of AMI}

Rapid diagnosis of acute myocardial infarction (AMI) is crucial. Current guidelines recommend measurement of troponin (Tn) - a marker of myocardial necrosis-in the detection of AMI.

Commercially available assays for $\mathrm{Tn}$ are now highly sensitive and can detect low-level elevations in Tn concentrations, but many clinicians have questioned the clinical importance of these low-level elevations. In a prospective substudy of the MERLIN-TIMI 36 trial, Marc Bonaca and colleagues thus tested the prognostic performance of a commercially available TnI assay in patients with suspected acute coronary syndromes.

The investigators used the ADVIA Centaur $^{\circledast}$ TnI-Ultra (Siemens Healthcare Diagnostics Inc., Deerfield, IL, USA) sandwich immunoassay, which has a detection limit of $0.006 \mu \mathrm{g} / \mathrm{l}$.

Compared with patients who had baseline TnI levels lower than the recommended $99^{\text {th }}$ percentile reference limit

$(0.04 \mu \mathrm{g} / \mathrm{l} ; n=1,589)$, those with

baseline $\mathrm{TnI} \geq 0.04 \mu \mathrm{g} / \mathrm{l}(n=2,924)$ had higher rates of death or MI at 30 days and at 12 months. Importantly, at both time points, the 319 patients who had baseline TnI levels between $0.04 \mu \mathrm{g} / \mathrm{l}$ and $0.1 \mu \mathrm{g} / \mathrm{l}$ (that is, low-level elevation) had a higher incidence of death or MI than the patients with baseline $\mathrm{TnI}<0.04 \mu \mathrm{g} / \mathrm{l}$. "Our findings ... establish the clinical relevance of such low-level increases in TnI using a currentgeneration assay at the contemporary cut point $\left(99^{\text {th }}\right.$ percentile) recommended by the universal definition of MI," remark the researchers.

Despite being undeniably useful in the diagnosis of AMI, markers of myocardial necrosis are only measurable after cell disintegration. To improve rapid AMI diagnosis, Till Keller and colleagues thought it might be prudent to measure markers of processes that are independent of myocardial necrosis. They thus tested prospectively whether measurement of copeptin-a marker of post-AMI remodeling_adds diagnostic information to Tn measurement.

Median copeptin levels (measured using the CT-proAVP LIA, BRAHMS AG, Henningsdorf, Germany; detection limit $0.4 \mathrm{pmol} / \mathrm{l}$ ) were highest within the first $3 \mathrm{~h}$ from onset of chest pain, whereas median TnT levels (measured using the Elecsys ${ }^{\circledR} 2010$ system, Roche Diagnostics, Mannheim, Germany; detection limit $0.01 \mu \mathrm{g} / \mathrm{l})$ were highest $12 \mathrm{~h}$ after chestpain onset. Assessment of copeptin in combination with TnT provided higher diagnostic power for AMI than assessment of TnT or copeptin alone, and than assessment of two other necrosis markers, myoglobin and CK-MB, alone or in combination with TnT.

Current-generation Tn assays have higher sensitivity than older assays in the identification of AMI within $3 \mathrm{~h}$ of chestpain onset. As seen for TnT measurement, however, assessment of copeptin in

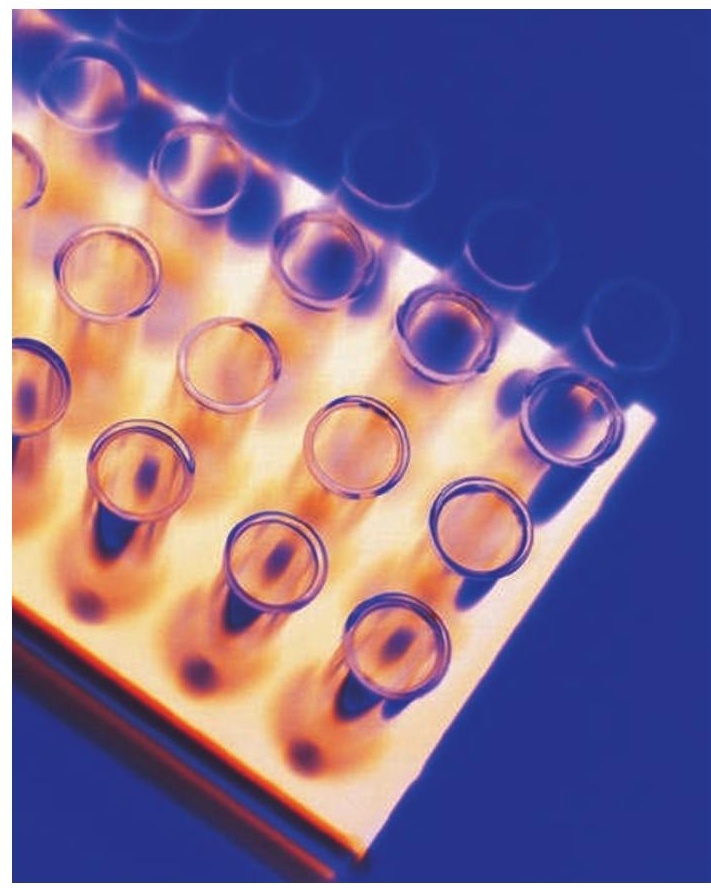

combination with TnI (using the same assay as Bonaca et al.) also provided higher diagnostic power for AMI than assessment of TnI alone and that of copeptin alone.

On the basis of their detailed analysis, Keller et al. conclude that "copeptin is not able to replace Tn in final rule-in of AMI, but allows earlier decision-making in clinical practice".

Bryony M. Mearns

Original articles Bonaca, M. et al. Prospective evaluation of the prognostic implications of improved assay performance with a sensitive assay for cardiac troponin I. J. Am. Coll. Cardiol. 55, 2118-2123 (2010) | Keller, T. et al. Copeptin improves early diagnosis of acute myocardial infarction. J. Am. Coll. Cardiol. 55, 2096-2106 (2010) 